\title{
Involvement of the unfolded protein response in the protective effects of growth hormone releasing hormone antagonists in the lungs
}

\author{
Mohammad S. Akhter ${ }^{1} \cdot$ Mohammad A. Uddin $^{1} \cdot$ Andrew V. Schally $^{2,3} \cdot$ Khadeja-Tul Kubra $^{1} \cdot$ Nektarios Barabutis $^{1}$ (B)
}

Received: 27 October 2020 / Accepted: 1 November 2020 / Published online: 13 November 2020

(c) The International CCN Society 2020

\begin{abstract}
Growth hormone releasing hormone (GHRH) antagonists enhance endothelial barrier function and counteract the LPSinduced lung endothelial hyperpermeability, the cardinal feature of the acute respiratory distress syndrome (ARDS). The unfolded protein response (UPR) is a multifaceted molecular mechanism, strongly involved in tissue defense against injury. The current study introduces the induction of UPR by GHRH antagonists, since those peptides induced several UPR activation markers, including the inositol-requiring enzyme-1 $\alpha$ (IRE1 $\alpha$ ), the protein kinase RNA-like ER kinase (PERK), and the activating transcription factor 6 (ATF6). On the other hand, the GHRH agonist MR-409 exerted the opposite effects. Furthermore, GHRH antagonists counteracted the kifunensine (UPR suppressor)-induced lung endothelial barrier dysfunction. Our observations suggest that UPR mediates, at least in part, the protective effects of GHRH antagonists in the lung microvasculature. To the best of our knowledge; this is the first study to provide experimental evidence in support of the hypothesis that UPR induction is a novel mechanism by which GHRH antagonists oppose severe human disease, including ARDS.
\end{abstract}

Keywords P53 $\cdot$ Lung injury $\cdot$ Endothelium $\cdot$ Vascular barrier $\cdot$ Inflammation

\section{Introduction}

Growth hormone releasing hormone (GHRH) is a hypothalamic hormone which regulates the release of growth hormone $(\mathrm{GH})$ from the anterior pituitary gland. The effects of GHRH are mediated by the pituitary type GHRH receptor (pGHRH-R), primarily expressed in the somatotrophs of the pituitary gland. Active splice variants of the human GHRH$\mathrm{R}$ have been identified in a diverse variety of human tissues, suggesting that the effects of GHRH in human physiology are not limited to the hypothalamic-pituitary-somatotropic axis (Barabutis 2020b).

Nektarios Barabutis

barabutis@ulm.edu

1 School of Basic Pharmaceutical and Toxicological Sciences, College of Pharmacy, University of Louisiana Monroe, 1800 Bienville Drive, Monroe, LA 71201, USA

2 Endocrine, Polypeptide, and Cancer Institute, Veterans Affairs Medical Center, Miami, FL, USA

3 Divisions of Medical Oncology and Endocrinology, Department of Medicine and Department of Pathology, Miller School of Medicine, University of Miami, Miami, FL, USA
GHRH possesses growth factor activities in cancers, since the activation of the GHRH receptor by this neuropeptide induces cancer promotion and metastasis; both in vivo and in vitro (Barabutis 2020a). The silencing of the intrinsic GHRH expression in human lung, prostate and breast cancers strongly suppresses their proliferation (Barabutis and Schally 2008). GHRH antagonists block the effects of GHRH in both cancerous and non-cancerous cell lines (Barabutis and Siejka 2020). The protective effects of those compounds in human tissues have been associated with strong anti-inflammatory and anti-oxidative properties (Schally et al. 2019).

GHRH antagonists suppress major inflammatory pathways, including the extracellular signal-regulated kinases 1/2 (ERK1/2) (Barabutis et al. 2010) and Janus kinase 2/ signal transducers and activators of transcription 3 (JAK2/ STAT3) (Siejka et al. 2010). Furthermore, those peptides reduce the generation of the reactive oxygen and nitrogen species, the expression of the inducible nitric oxide synthase (iNOS), as well as the levels of protein and lipid oxidation (Barabutis et al. 2011, 2020). In bovine pulmonary artery endothelial cells (BPAECs), the GHRH antagonist MIA-602 induced P53 expression, suppressed F-actin fibers, and deactivated the actin-severing activity of cofilin. Those 
events resulted to the endothelial barrier enhancement. On the other hand, the GHRH agonist MR-409 increased the lung endothelial permeability, by counteracting the previous MIA-602-triggered effects. Vascular barrier dysfunction and pulmonary edema is the cardinal feature of the acute respiratory distress syndrome (ARDS), including the ARDS related to SARS-CoV-2 (Hariri and Hardin 2020; Romagnoli et al. 2020; Uddin et al. 2019b).

UPR senses cellular stresses, to devise carefully orchestrated tissue repairing processes. It consists of the protein kinase RNA-like ER kinase (PERK), the activating transcription factor 6 (ATF6), and the inositol-requiring enzyme-1 $\alpha$ (IRE1 $\alpha)$. Those elements are closely related to the binding immunoglobulin protein (BiP), which is released upon endoplasmic reticulum stress increases (Barabutis 2020d). Moreover, the protein disulfide isomerase (PDI) and endoplasmic reticulum oxidoreductin 1- $\mathrm{L}$ alpha (ERO1-L $\alpha$ ) are markers of UPR induction. UPR activation induces P53 (Akhter et al. 2019). The goal of the present study is to introduce the effects of GHRH analogs in UPR activation, as it relates to the vascular barrier function. The new information will most probably reveal new investigative avenues in the development of medical countermeasures against lung inflammatory disease, including ARDS.

\section{Materials and methods}

\section{Reagents}

Kifunensine (KIF) (IC15995201), anti-rabbit IgG HRPlinked antibody (95017-556), nitrocellulose membranes (10063-173) and RIPA buffer (AAJ63306-AP) were obtained from VWR (Radnor, PA). The IRE1 $\alpha$ (3294), phospho-PERK (3179), PERK (5683), ATF6 (65880), BiP (3183), PDI (2446), ERO1-L $\alpha$ (3264), phospho-MLC2 (3674), MLC2 (3672), phospho-cofilin (3313) and cofilin (3318) antibodies were purchased from Cell Signaling (Danvers, MA). The $\beta$-actin antibody (A5441) was obtained from Sigma-Aldrich (St Louis, MO). The phospho-IRE1 $\alpha$ antibody (PA1-16927) was purchased from Thermo Fisher Scientific (Waltham, MA), and the GHRH antagonist Acetyl(D-Arg ${ }^{2}$ )-GRF (1-29) amide (human) trifluoroacetate salt (4030691) from Bachem (Torrance, CA). The peptides MIA602 and MR-409 were synthesized in AVS laboratory.

\section{Cell culture}

BPAECs (PB30205) were purchased from Genlantis (San Diego, CA). The cells were cultured in DMEM medium supplemented with $10 \%$ fetal bovine serum, and $1 \mathrm{X}$ penicillin/ streptomycin. Cultures were kept in a humidified atmosphere of $5 \% \mathrm{CO}_{2} / 95 \%$ air and $37{ }^{\circ} \mathrm{C}$ temperature.

\section{Measurement of endothelial barrier function}

The barrier function of the endothelial monolayer was estimated by the electric cell-substrate impedance sens-

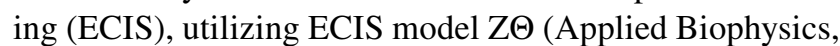
Troy, NY, USA) (Akhter et al. 2020a).

\section{Western blot analysis}

Proteins were extracted from cells using RIPA buffer and were separated by electrophoresis on $8 \%$ sodium dodecyl sulfate (SDS-PAGE) Tris-HCl gels. Those proteins were transferred onto nitrocellulose membranes, and were incubated at room temperature in a solution of 5\% non-fat dry milk in Tris-buffered saline for an hour. Those membranes were exposed overnight to the appropriate primary antibodies $(1: 1000)$ at $4{ }^{\circ} \mathrm{C}$. Next day, the blots were incubated with the corresponding secondary antibodies $(1: 2000)$ to develop signals for the immunoreactive proteins. Those signals were visualized in a ChemiDoc ${ }^{\mathrm{TM}}$ Touch Imaging System from Bio-Rad (Hercules, CA). The $\beta$-actin was used as a loading control unless indicated otherwise.

\section{Densitometry and statistical analysis}

Image $\mathbf{J}$ software (NIH) was used to perform densitometry of immunoblots. All data are expressed as mean \pm SEM (standard error of mean). Student's $t$ test was used to determine statistically significant differences among the groups. A value of $P<0.05$ was considered significant. GraphPad Prism (version 5.01) was used to analyze data. The letter $\mathrm{n}$ represents the number of experimental repeats.

\section{Results}

\section{Regulation of IRE1 a by GHRH analogs in the lungs}

BPAECs were treated with vehicle (0.01\% DMSO), or MIA-602 $(0.5,1 \mu \mathrm{M})$, or MR-409 $(0.5,1 \mu \mathrm{M})$ for $8 \mathrm{~h}$. The results demonstrate that MIA-602 significantly increased the phosphorylation of IRE1 $\alpha$. In contrast, MR-409 suppressed IRE1 $\alpha$ phosphorylation (Fig. 1a).

\section{Regulation of PERK by GHRH analogs in the lungs}

The bovine cells were treated with vehicle $(0.01 \%$ DMSO $)$, or MIA-602 $(0.5,1 \mu \mathrm{M})$, or MR-409 $(0.5,1 \mu \mathrm{M})$. MIA-602 significantly induced the activation of PERK at both doses 

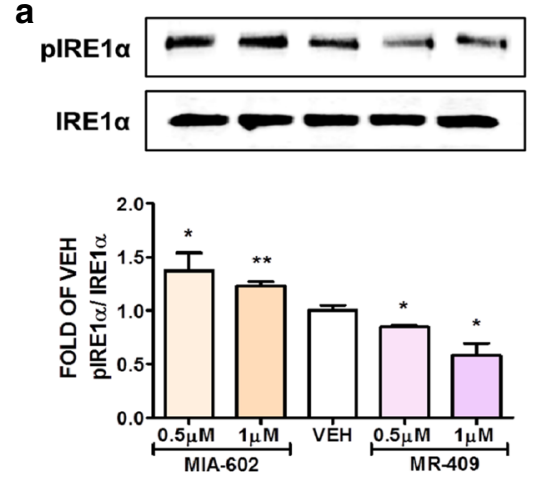

d
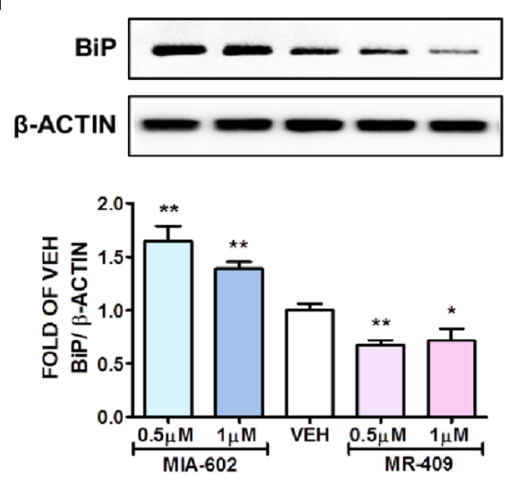

g

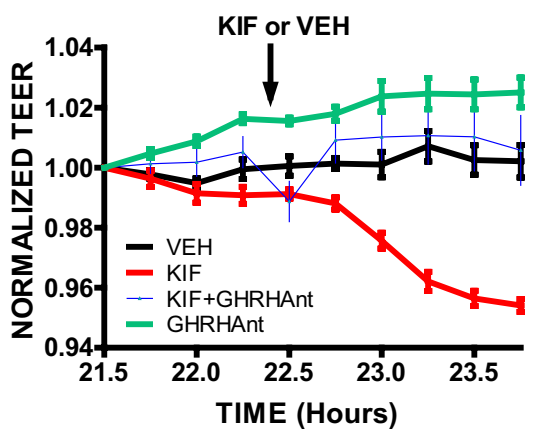

b
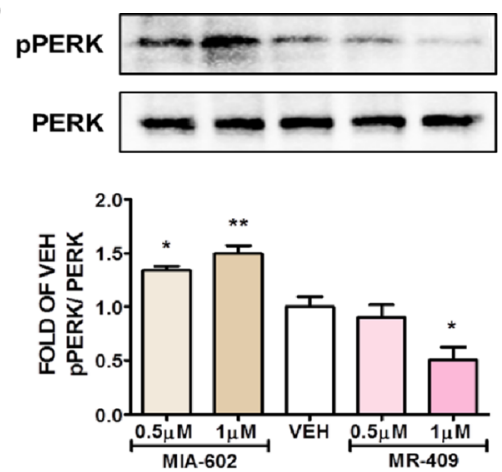

e
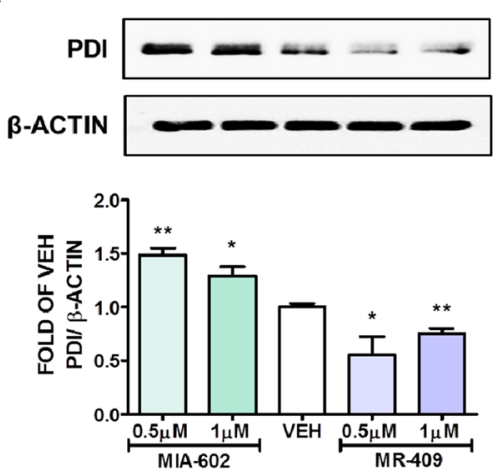

h
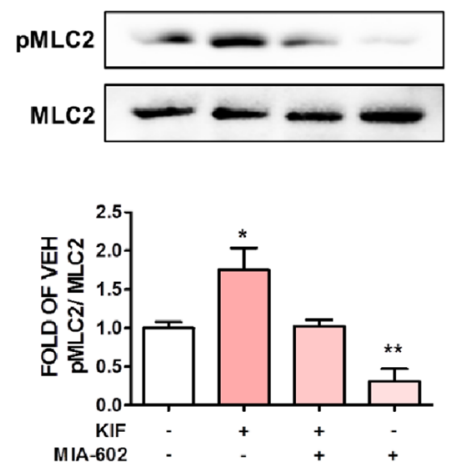

c
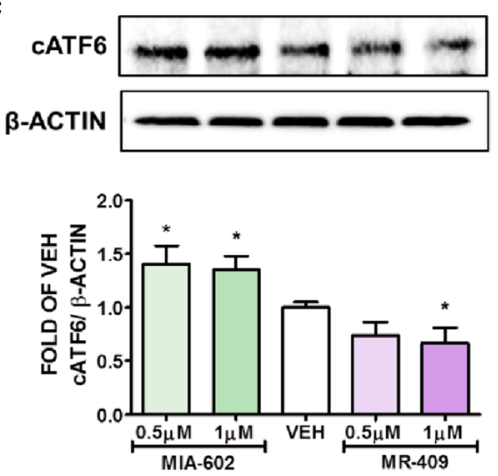

f
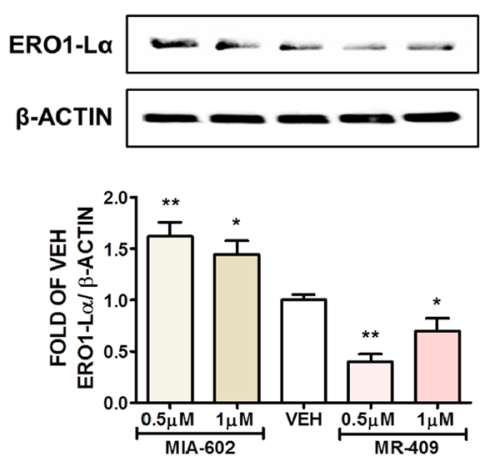

i
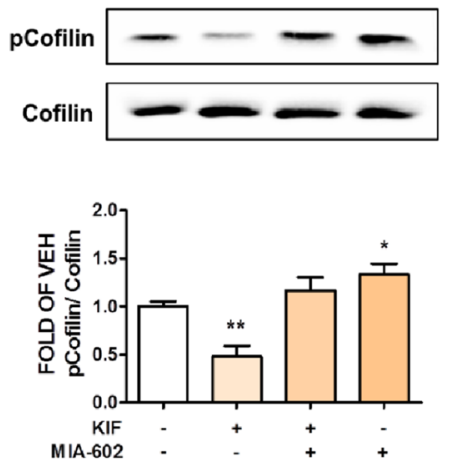

Fig. 1 Effects of GHRH analogs on UPR activation and lung endothelial barrier function: Western blot analysis of a pIRE1 $\alpha$ and IRE $1 \alpha, \mathbf{b}$ pPERK and PERK, $\mathbf{c}$ cATF6 and $\beta$-actin, $\mathbf{d}$ BiP and $\beta$-actin, e PDI and $\beta$-actin, and $\mathbf{f}$ ERO1-L $\alpha$ and $\beta$-actin after treatment of BPAECs with either vehicle (0.01\% DMSO), or MIA-602 $(0.5 \mu \mathrm{M}$ and $1 \mu \mathrm{M})$, or MR-409 $(0.5 \mu \mathrm{M}$ and $1 \mu \mathrm{M})$ for $8 \mathrm{~h}$. Each blot represents 3 independent experiments. The signal intensity of pIRE $1 \alpha$, pPERK, cATF6, BiP, PDI, and ERO1-L $\alpha$ bands was analyzed by densitometry. Protein levels were normalized to $\beta$-actin, unless stated otherwise in the signal intensity graph. $* P<0.05$, $* * P<0.01$ versus vehicle. Mean \pm SEM. g Confluent monolayers of BPAEC were pre-treated with either vehicle (0.01\% DMSO) or GHRH antagonist (GHRHAnt) $(1 \mu \mathrm{M})$ for $8 \mathrm{~h}$, followed by treatment with either vehicle $(0.01 \% \mathrm{DMSO})$ or $25 \mu \mathrm{M}$ of KIF (black arrow). A gradual increase in TEER values (decreased permeability) was observed in the cells treated with the GHRH antagonist (green line). Those cells treated with KIF (red line) exerted a gradual decrease in their TEER values (increased permeability). However, those lung cells that were pretreated with the GHRH antagonist were protected against the KIFinduced barrier dysfunction (blue line). $\mathrm{N}=3$, Mean $\pm \mathrm{SE}$. Western blot analysis of $\mathbf{h}$ pMLC2 and MLC2, i pCofilin and Cofilin. BPAECs were pre-treated with either vehicle $(0.01 \%$ DMSO) or kifunensine (KIF) $(2 \mu \mathrm{M})$ for $24 \mathrm{~h}$, and consequently treated with either vehicle $(0.01 \%$ DMSO) or MIA-602 $(1 \mu \mathrm{M})$ for $8 \mathrm{~h}$. Each blot represents 3 independent experiments. The signal intensity of protein bands was analyzed by densitometry. Protein levels of pMLC 2 and pCofilin were normalized MLC2 and Cofilin respectively. $* P<0.05$, $* * P<0.01$ versus vehicle. Mean \pm SEM 
after $8 \mathrm{~h}$ of treatment. Conversely, PERK activation was suppressed by MR-409 (Fig. 1b).

\section{Regulation of ATF6 by GHRH analogs in the lungs}

BPAECs were treated with vehicle (0.01\% DMSO), or MIA$602(0.5,1 \mu \mathrm{M})$ or MR-409 $(0.5,1 \mu \mathrm{M})$ for $8 \mathrm{~h}$. MIA-602 induced the cATF6 expression levels, while MR-409 exerted the opposite effects (Fig. 1c).

\section{Regulation of BiP, PDI and ER01-La by GHRH analogs in the lungs}

Lung cells were treated with vehicle $(0.01 \%$ DMSO), or MIA-602 $(0.5,1 \mu \mathrm{M})$, or MR-409 $(0.5,1 \mu \mathrm{M})$. The results demonstrate that the GHRH antagonist MIA-602 significantly enhanced the UPR markers BiP (Fig. 1d), PDI (Fig. 1e) and ERO1-L $\alpha$ (Fig. 1f) after $8 \mathrm{~h}$ of treatment. On the other hand, MR-409 reduced the UPR levels, as reflected in the expression of all three markers.

\section{GHRH antagonists protect against kifunensine (KIF)-induced lung endothelial hyperpermeability}

Confluent monolayers of BPAECs were pre-treated with vehicle $(0.01 \%$ DMSO) or GHRH antagonist $(1 \mu \mathrm{M})$ for $8 \mathrm{~h}$, and then treated with vehicle (0.01\% DMSO) or KIF $(25 \mu \mathrm{M})$. GHRH antagonist increased the transendothelial resistance (TEER) (decreased permeability) of those cells. On the other hand, KIF reduced their TEER, indicated hyper-permeability responses (Fig. 1g). Those cells that were pre-treated with the GHRH antagonist were protected against the KIF-induced endothelial hyper-permeability. Moreover, BPAECs were treated with vehicle $(0.01 \%$ DMSO) or KIF $(2 \mu \mathrm{M})$ for $24 \mathrm{~h}$ prior to vehicle $(0.01 \%$ DMSO) or the GHRH antagonist MIA-602 $(1 \mu \mathrm{M})$ exposure $(8 \mathrm{~h})$. MIA-602 significantly reduced the KIF-induced phosphorylation of MLC2 (Fig. 1h), and suppressed the activation (de-phosphorylation) of cofilin by KIF (Fig. 1i).

\section{Discussion}

UPR activation exerts a prominent role in the maintenance of the pulmonary (Akhter et al. 2020a; Barabutis 2020d) and cardiovascular system (Hetz et al. 2019; Kubra et al. 2020a). PERK-knockout mice significantly exacerbate the transverse aortic constriction (TAC)-induced lung vascular remodeling and lung fibrosis (Liu et al. 2014), while the impaired (mutated) form of BiP causes abnormal secretion of the pulmonary surfactant. Mice expressing mutant $\mathrm{BiP}$ were subjected to respiratory failure due to reduced expression of surfactant protein C (Mimura et al. 2007). We have recently shown that the UPR suppressor Kifunensine weakened the endothelial barrier function in a dose- and timedependent manner (Akhter et al. 2020a).

Moreover, it was speculated that the protective activities of the heat shock protein 90 (Hsp90) inhibitors against the LPS-induced lung injury are at least partially mediated due to UPR activation. The Hsp90 inhibitors AUY-922 and 17-AAG induced the expression of all three UPR branches (IRE1 $\alpha$, PERK and ATF6) in bovine and mice lung cells both in vivo and in vitro (Kubra et al. 2020b; Uddin et al. 2020). Hence, it was suggested that the anti-inflammatory activities of those compounds in the lung microvasculature are associated to UPR induction. Later on it was revealed that the AUY-922 opposed the kifunensine (UPR suppressor)-induced lung endothelial hyperpermeability, suggesting that UPR modulation is a master regulator of vascular barrier function (Kubra et al. 2020c).

UPR mediates a wide variety of signaling processes including ER-associated degradation (ERAD), apoptosis and autophagy, dictating cellular fate during stress condition (Akhter et al. 2020c). Our results demonstrate that the GHRH antagonist MIA-602 induced the activation of IRE1 $\alpha$ (Fig. 1a), PERK (Fig. 1b) and ATF6 (Fig. 1c), while the GHRH agonist MR-409 exerted the opposite effects (Fig. 1a, b, c). Furthermore, we evaluated the expression of BiP (Fig. 1d), ERO1-L $\alpha$ (Fig. 1f) and PDI (Fig. 1e) in cells treated with MIA-602 or the GHRH agonist MR-409. ERO1-L $\alpha$ catalyzes the formation of disulfide bonds into the ER resident proteins, and PDI is a redox ER chaperone which acts to repair misfolded proteins (Barabutis 2020d). It appears that MIA-602 induced BiP, PDI and ERO1-La, while the GHRH agonist MR-409 exerted the opposite effects.

P53 is a tumor suppressor protein which regulates essential biological processes, including cell cycle arrest, DNA repair, senescence and apoptosis. It also supports the vasculature against inflammation by suppressing the inflammatory apurinic/apyrimidinic endonuclease $1 /$ redox effector factor 1 (APE1/Ref1) (Uddin et al. 2019a), RhoA (Barabutis et al. 2018), NF-kB (Barabutis 2020c), and lipid peroxidation (Akhter et al. 2020b). Moreover this transcription factor (P53) sabotaged the actin severing activity of cofilin (Barabutis et al. 2018). Recent observations report that GHRH antagonists are P53 inducers and vascular barrier enhancers (Uddin et al. 2019b).

Cofilin is an actin-binding protein that depolymerizes filamentous actin (F-actin), and generates free filaments that are accessible to globular actin (G-actin). Activated cofilin reduces tight junction proteins, and increases permeability (Kubra et al. 2020a). The present study provides evidence that GHRH antagonists significantly decreased the activation of cofilin due to kifunensine treatment (Fig. 1i). Moreover, MIA-602 suppressed the kifunensine-induced phosphorylation of myosin light chain 2 (MLC2) (Fig. 1h), and opposed 
the kifunensine-induced endothelial barrier dysfunction (Fig. 1g). Phosphorylated MLC2 induces the formation of F-actin fiber, increasing the endothelial permeability. Remarkably, lung cells treated with the GHRH antagonist (GHRHAnt) were protected against the kifunensine-induced lung endothelial barrier dysfunction, as indicated by measurements of transendothelial resistance (TEER) (Fig. 1g).

To the best of our knowledge, this is the first study to provide experimental evidence in support of the hypothesis that UPR mediates the protective activities of GHRH antagonists in the lung microvasculature. Future studies will delineate the exact UPR branches which mediate the protective effects of those analogs in a diverse variety of human tissues, including the lungs.

Funding The study was supported by I) R\&D, Research Competitiveness Subprogram (RCS) of the Louisiana Board of Regents through the Board of Regents Support Fund (LEQSF(2019-22)-RD-A-26) (PI: N.B), the Faculty Research Support Program from the Dean's office (ULM) (PI: N.B), and the NIH/NIGMS (5 P20 GM103424-15, 3 P20 GM103424-15S1). AVS is supported by the Medical Research Service of the Department of Veterans Affairs, and University of Miami Miller School of Medicine.

\section{Compliance with ethical standards}

Conflict of interest The authors declare no conflicts of interest.

\section{References}

Akhter MS, Uddin MA, Barabutis N (2019) Unfolded protein response regulates P53 expression in the pulmonary endothelium. J Biochem Mol Toxicol 33:e22380. https://doi.org/10.1002/jbt.22380

Akhter MS, Kubra KT, Uddin MA, Barabutis N (2020a) Kifunensine compromises lung endothelial barrier function. Microvasc Res 132:104051. https://doi.org/10.1016/j.mvr.2020.104051

Akhter MS, Uddin MA, Barabutis N (2020b) P53 regulates the redox status of lung endothelial cells. Inflammation 43:686-691. https:// doi.org/10.1007/s10753-019-01150-7

Akhter MS, Uddin MA, Kubra KT, Barabutis N (2020c) Autophagy, unfolded protein response and lung disease. Curr Res Cell Biol 1:100003. https://doi.org/10.1016/j.crcbio.2020.100003

Barabutis N (2020a) A glimpse at growth hormone-releasing hormone cosmos. Clin Exp Pharmacol Physiol 47:1632-1634. https://doi. org/10.1111/1440-1681.13324

Barabutis N (2020b) Growth hormone releasing hormone in the unfolded protein response context. Endocrine 67:291-293. https://doi. org/10.1007/s12020-020-02205-8

Barabutis N (2020c) P53 in acute respiratory distress syndrome. Cell Mol Life Sci. https://doi.org/10.1007/s00018-020-03629-1

Barabutis N (2020d) Unfolded protein response in lung health and disease. Front Med (Lausanne) 7:344. https://doi.org/10.3389/ fmed.2020.00344

Barabutis N, Schally AV (2008) Knocking down gene expression for growth hormone-releasing hormone inhibits proliferation of human cancer cell lines. Br J Cancer 98:1790-1796. https://doi.org/10.1038/ sj.bjc. 6604386

Barabutis N, Siejka A (2020) The highly interrelated GHRH, p53, and Hsp90 universe. Cell Biol Int 44:1558-1563. https://doi. org/10.1002/cbin.11356
Barabutis N, Siejka A, Schally AV, Block NL, Cai R, Varga JL (2010) Activation of mitogen-activated protein kinases by a splice variant of GHRH receptor. J Mol Endocrinol 44:127-134. https://doi. org/10.1677/JME-09-0121

Barabutis N, Siejka A, Schally AV (2011) Growth hormone releasing hormone induces the expression of nitric oxide synthase. J Cell Mol Med 15:1148-1155. https://doi.org/10.111 1/j.1582-4934.2010.01096.x

Barabutis N, Dimitropoulou C, Gregory B, Catravas JD (2018) Wild-type p53 enhances endothelial barrier function by mediating RAC1 signalling and RhoA inhibition. J Cell Mol Med 22:1792-1804. https ://doi.org/10.1111/jcmm.13460

Barabutis N, Akhter MS, Uddin MA, Kubra KT, Schally AV (2020) GHRH antagonists protect against hydrogen peroxide-induced breakdown of brain microvascular endothelium integrity. Horm Metab Res 52:336-339. https://doi.org/10.1055/a-1149-9347

Hariri L, Hardin CC (2020) Covid-19, angiogenesis, and ARDS endotypes. N Engl J Med 383:182-183. https://doi.org/10.1056/NEJMe 2018629

Hetz C, Axten JM, Patterson JB (2019) Pharmacological targeting of the unfolded protein response for disease intervention. Nat Chem Biol 15:764-775. https://doi.org/10.1038/s41589-019-0326-2

Kubra KT, Akhter MS, Uddin MA, Barabutis N (2020a) Unfolded protein response in cardiovascular disease. Cell Signal 73:109699. https:// doi.org/10.1016/j.cellsig.2020.109699

Kubra KT, Uddin MA, Akhter MS, Barabutis N (2020b) Hsp90 inhibitors induce the unfolded protein response in bovine and mice lung cells. Cell Signal 67:109500. https://doi.org/10.1016/j.cellsig.2019.10950 0

Kubra KT, Uddin MA, Akhter MS, Barabutis N (2020c) Luminespib counteracts the kifunensine-induced lung endothelial barrier dysfunction. Curr Res Toxicol 1:111-115. https://doi.org/10.1016/j. crtox.2020.09.003

Liu X et al (2014) Endoplasmic reticulum stress sensor protein kinase R-like endoplasmic reticulum kinase (PERK) protects against pressure overload-induced heart failure and lung remodeling. Hypertension 64:738-744. https://doi.org/10.1161/HYPERTENSIONAHA .114 .03811

Mimura N et al (2007) Aberrant quality control in the endoplasmic reticulum impairs the biosynthesis of pulmonary surfactant in mice expressing mutant BiP. Cell Death Differ 14:1475-1485. https://doi. org/10.1038/sj.cdd.4402151

Romagnoli S, Peris A, De Gaudio AR, Geppetti P (2020) SARS-CoV-2 and COVID-19: from the bench to the bedside. Physiol Rev 100:1455-1466. https://doi.org/10.1152/physrev.00020.2020

Schally AV, Zhang X, Cai R, Hare JM, Granata R, Bartoli M (2019) Actions and potential therapeutic applications of growth hormonereleasing hormone agonists. Endocrinology 160:1600-1612. https ://doi.org/10.1210/en.2019-00111

Siejka A, Schally AV, Barabutis N (2010) Activation of Janus kinase/ signal transducer and activator of transcription 3 pathway by growth hormone-releasing hormone. Cell Mol Life Sci 67:959-964. https:// doi.org/10.1007/s00018-009-0224-y

Uddin MA, Akhter MS, Siejka A, Catravas JD, Barabutis N (2019a) P53 supports endothelial barrier function via APE1/Ref1 suppression. Immunobiology 224:532-538. https://doi.org/10.1016/j.imbio 2019.04.008

Uddin MA, Akhter MS, Singh SS, Kubra KT, Schally AV, Jois S, Barabutis N (2019b) GHRH antagonists support lung endothelial barrier function. Tissue Barriers 7:1669989. https://doi.org/10.1080/21688 370.2019.1669989

Uddin MA, Kubra KT, Sonju JJ, Akhter MS, Seetharama J, Barabutis N (2020) Effects of heat shock protein 90 inhibition in the lungs. Med Drug Discov 6:100046. https://doi.org/10.1016/j.medid d.2020.100046 